\title{
TRPV1 Channels Regulate Cortical Excitability in Humans
}

\author{
Francesco Mori, ${ }^{1,3}$ Michele Ribolsi, ${ }^{2}$ Hajime Kusayanagi, ${ }^{1,3}$ Fabrizia Monteleone, ${ }^{1,3}$ Vilma Mantovani, ${ }^{4}$ Fabio Buttari, ${ }^{1,3}$ \\ Elena Marasco, ${ }^{4}$ Giorgio Bernardi, ${ }^{1,3}$ Mauro Maccarrone, ${ }^{3,5}$ and Diego Centonze ${ }^{1,3}$ \\ Centers for ${ }^{1}$ Neurology and ${ }^{2}$ Psychiatry, Department of Neuroscience, University Tor Vergata, 00133 Rome, Italy, ${ }^{3}$ Santa Lucia Foundation/European \\ Center for Brain Research, 00143 Rome, Italy, ${ }^{4}$ Applied Biomedical Research Center, S. Orsola-Malpighi Polyclinic, 40138 Bologna, Italy, and ${ }^{5}$ Department \\ of Biomedical Sciences, University of Teramo, 64100 Teramo, Italy
}

Studies in rodents show that transient receptor potential vanilloid 1 (TRPV1) channels regulate glutamate release at central and peripheral synapses. In humans, a number of nonsynonymous single-nucleotide polymorphisms (SNPs) have been described in the TRPV1 gene, and some of them significantly alter the functionality of the channel. To address the possible role of TRPV1 channels in the regulation of synaptic transmission in humans, we studied how TRPV1 genetic polymorphisms affect cortical excitability measured with transcranial magnetic stimulation (TMS). Two SNPs of the TRPV1 gene were selected and genotyped (rs222747 and rs222749) in a sample of 77 healthy subjects. In previous cell expression studies, the " $G$ " allele of rs222747 was found to enhance the activity of the channel, whereas rs222749 had no functional effect. Allelic variants in the rs222749 region were not associated with altered cortical response to single, paired, and repetitive TMS. In contrast, subjects homozygous for the G allele in rs222747 exhibited larger short-interval intracortical facilitation (a measure of glutamate transmission) explored through paired-pulse TMS of the primary motor cortex. Recruitment curves, short-interval intracortical inhibition, intracortical facilitation, and long-interval intracortical inhibition were unchanged. LTPand LTD-like plasticity explored through intermittent or continuous theta-burst stimulation was also similar in the " $G$ " and "non-G" subjects. To our knowledge, our results provide the first evidence that TRPV1 channels regulate cortical excitability to paired-pulse stimulation in humans.

\section{Introduction}

Transient receptor potential vanilloid 1 (TRPV1) channels are nonselective cation channels activated by a variety of chemical and physical stimuli, which include vanilloid compounds, voltage, heat, protons, and lipid molecules, such as endocannabinoids and eicosanoids (Van Der Stelt and Di Marzo, 2004, 2005; De Petrocellis and Di Marzo, 2005; Suh and Oh, 2005; Pingle et al., 2007; Di Marzo and De Petrocellis, 2010).

Studies in animals show that these channels are involved in the regulation of synaptic transmission in peripheral (Caterina et al., 1997; Sikand and Premkumar, 2007) and central (Gibson et al., 2008; Peters et al., 2010; Shoudai et al., 2010) structures, by enhancing glutamate release from nerve endings. Accordingly, physiological studies have shown that stimulation of TRPV1 channels with capsaicin or anandamide enhances the frequency of glutamate-mediated spontaneous and miniature EPSCs, whereas GABAergic synaptic transmission is unaffected (Yang et al., 1998; Marinelli et al., 2002, 2003; Li et al., 2004; Derbenev et al., 2006; Starowicz et al., 2007; Xing and Li, 2007; Musella et al.,

\footnotetext{
Received May 20, 2011; revised Nov. 12, 2011; accepted Nov. 18, 2011.

Author contributions: G.B. and D.C. designed research; F.Mor., M.R., H.K., F.Mon., V.M., F.B., and M.M. performed research; M.R., V.M., E.M., and M.M. analyzed data; F.Mor. and D.C. wrote the paper.

This study was supported by a grant from the Italian Ministry of Health (D.C.) and the Tercas Foundation (M.M., D.C.).

The authors declare no competing financial interests.

Correspondence should be addressed to Diego Centonze, Clinica Neurologica, Dipartimento di Neuroscienze, Universit à Tor Vergata, Via Montpellier 1, 00133 Rome, Italy. E-mail: centonze@uniroma2.it.

DOI:10.1523/JNEUROSCI.2531-11.2012

Copyright $\odot 2012$ the authors $\quad 0270-6474 / 12 / 320873-07 \$ 15.00 / 0$
}

2009). Recent studies also show that the regulation of spontaneous glutamate release by TRPV1 receptors is activity dependent (Peters et al., 2010; Shoudai et al., 2010), because afferent activation triggers long-lasting asynchronous glutamate release only from synapses expressing the TRPV1 receptor, strongly potentiating the duration of postsynaptic spiking (Peters et al., 2010). TRPV1 has also been involved in the regulation of synaptic plasticity and in particular in hippocampal long-term potentiation (LTP) (Marsch et al., 2007) and depression (LTD) (Gibson et al., 2008) and also in hippocampus-dependent learning in mice (Marsch et al., 2007).

A number of nonsynonymous single-nucleotide polymorphisms (SNPs) have been described in the human TRPV1 gene, and data obtained by transfection of HEK cells with DNA containing the human SNP show that some of them confer specific functional properties to TRPV1 channels (Xu et al., 2007). SNP rs222747, in particular, is localized in the region of the ankyrin repeat domains, and its variant is associated with greater maximal response to capsaicin and to anandamide (Xu et al., 2007). It also increases TRPV1 mRNA and protein expression in cell surface, providing convincing evidence that subjects bearing this variant are characterized by enhanced functionality of the channel (Xu et al., 2007). On the contrary, other nonsynonymous SNP variants, such as rs222749, do not cause substantial effects on the pharmacological and expression properties of the TRPV1 channel (Xu et al., 2007).

In the present study, the role of TRPV1 channels in the control of cortical excitability in humans was explored by comparing the response to single transcranial magnetic stimulation (TMS) and 
paired-pulse TMS, as well as to theta-burst stimulation (TBS), in subjects with the rs222747 or rs222749 SNP variants of the TRPV1 gene. It is worth mentioning that the role of TRPV1 channels in the control of synaptic transmission within the cerebral cortex has not been addressed previously even in animals.

\section{Materials and Methods}

The study was approved by the Ethics Committee of the University Hospital Tor Vergata (Rome, Italy).

Subjects and SNP genotyping. We submitted to SNP genotyping 550 healthy subjects, recruited among investigators, nurses, and nonbiological relatives of subjects hospitalized in the Neurology Department of the University Hospital Tor Vergata. All subjects were Italian natives, belonging to European Caucasian ethnicity. Among them, 77 right-handed (Oldfield, 1971) healthy volunteers (31 males; mean age, $38.3 \pm 10.2$ years) gave consent to the TMS study. All subjects gave written informed consent. Subjects and investigators were blinded to genotyping results during data collection and all TMS experimental procedures. All data were collected prospectively.

The MassARRAY Assay Design 3.1 software was used to design a single 20-multiplex reaction in which the two SNPs rs222747 and rs222749 of the TRPV1 gene were included. Genotyping was performed using iPLEX Gold technology (Jurinke et al., 2002) and MassARRAY high-throughput DNA analysis with matrix-assisted laser desorption/ionization time-of-flight mass spectrometry (Sequenom), according to the instructions of the manufacturer. The two TRPV1 SNPs showed a call rate $>95 \%$, with no significant departure from Hardy-Weinberg equilibrium.

TMS. Electromyographic traces were recorded from the first dorsal interosseous (FDI) muscles of the left hand with 9-mm-diameter, silversilver chloride surface cup electrodes. The active electrode was placed over the muscle belly, and the reference electrode was placed over the metacarpophalangeal joint of the index finger. Responses were amplified with a Digitimer D360 amplifier through filters set at $20 \mathrm{~Hz}$ and $2 \mathrm{kHz}$ with a sampling rate of $5 \mathrm{kHz}$ and then recorded by a computer with Signal software (Cambridge Electronic Devices).

Motor-evoked potentials (MEPs) were evoked through a figure-ofeight coil with external loop diameter of $70 \mathrm{~mm}$ connected to a Magstim $200^{2}$ magnetic stimulator (Magstim Company). The hand motor area of right primary motor cortex (M1) was defined as the point at which stimulation evoked the largest MEP from the contralateral FDI muscle. The motor hotspot was identified at the beginning of each experimental session and marked over the patient's scalp with a pencil. The coil was held tangentially to the scalp surface with the handle pointing posteriorly and laterally at $\sim 45^{\circ}$ with respect to the midsagittal axis of the head. We defined the resting motor threshold (RMT) as the lowest intensity that evoked five small responses $(\sim 50 \mu \mathrm{V})$ in the contralateral FDI muscle in a series of 10 stimuli when the subject kept the FDI muscles relaxed in both hands, according to international standards. Active motor threshold (AMT) was defined as the lowest intensity that evoked five small responses $(\sim 200 \mu \mathrm{V})$ in a series of 10 stimulations when the subject made $10 \%$ of maximal voluntary contraction (Rothwell, 1997).

MEP amplitudes elicited by single-pulse TMS of increasing intensities $(90,100,110,120,130,140$, and $150 \%$ of the RMT) were measured to calculate recruitment curves of M1. Ten pulses were delivered for each stimulus intensity.

Paired-pulse TMS. Paired-pulse TMS was used to assess short-interval cortical facilitation (SICF; believed to follow the activation of glutamatergic cortical interneurons) (Di Lazzaro et al., 2003), short-interval cortical inhibition/facilitation (SICI/ICF; mediated by intrinsic GABA $\mathrm{A}_{\mathrm{A}}$ ergic or excitatory circuits) (Kujirai et al., 1993; Ziemann, 2004; Florian et al., 2008), and long-interval intracortical inhibition (LICI; mediated by local $\mathrm{GABA}_{\mathrm{B}}$ pathways) (Valls-Solé et al., 1992; Werhahn et al., 1999) of the right $\mathrm{M} 1$.

To assess SICF, the conditioning stimulus (CS) was delivered at $90 \%$ of right RMT (Hanajima et al., 2002), following the test stimulus (TS) at interstimulus intervals (ISI) of $1.5,2.1,2.7,3.7$, and $4.5 \mathrm{~ms}$. Ten noncon-
Table 1. Allele frequencies of TRPV1 gene SNPs

\begin{tabular}{clccc}
\hline Alleles & Type & Total sample $(n=550)$ & Stimulated $(n=77)$ & HapMap \\
\hline rs222747 & & & & \\
CC & Wild-type & $279(50.5 \%)$ & 30 & $66.6 \%$ \\
CG & Heterozygote & $237(43.2 \%)$ & 35 & $30.0 \%$ \\
GG & Homozygote & $34(6.2 \%)$ & 12 & $3.1 \%$ \\
rs222749 & & & & \\
CC & Wild-type & $499(91.1 \%)$ & 58 & $91.4 \%$ \\
CT & Heterozygote & $46(8.4 \%)$ & 14 & $6.9 \%$ \\
TT & Homozygote & $5(0.7 \%)$ & 5 & $1.7 \%$ \\
\hline
\end{tabular}

The frequency genotype data are selected from the public database based on HapMap data for European-Americans (CEU population) (http://www.hapmap.org).

ditioned MEPs and 10 conditioned MEPs, at each intensity, were collected in randomized order at a rate of $0.2 \mathrm{~Hz}$.

To assess SICI/ICF, a subthreshold CS, delivered 2 and $3 \mathrm{~ms}$ (for SICI) or 10 and $15 \mathrm{~ms}$ (for ICF) before the TS, was used to preferentially excite M1 intracortical inhibitory or excitatory fibers, and the subsequent reduction or potentiation in contralateral MEP amplitude compared with the nonconditioned MEP provided a measure of SICI or ICF, respectively. The CS was delivered at $80 \%$ of right AMT (Kujirai et al., 1993; Rothwell, 1997; Peurala et al., 2008).

LICI was tested following the protocol adopted by Valls-Solé et al. (1992). The intensity of CS was set at 120\% RMT. Three conditions were presented in a random order: control (TS given alone) and two pairedpulse conditions (TS preceded by CS) at one of three different ISIs (50, 100 , and $150 \mathrm{~ms})$.

Measurements were made on each individual trial, and the mean peakto-peak amplitude of the conditioned MEP was expressed as a percentage of the mean peak-to-peak amplitude of the unconditioned test pulse.

TBS. Intermittent (iTBS) or continuous (cTBS) TBS were delivered over M1 through a Magstim Rapid ${ }^{2}$ stimulator. Stimulation intensity was $80 \%$ of AMT. The iTBS protocol consisted of 10 bursts, with each burst composed of three stimuli at $50 \mathrm{~Hz}$, repeated at a theta frequency of $5 \mathrm{~Hz}$ every $10 \mathrm{~s}$ for a total of 600 stimuli $(200 \mathrm{~s})$. cTBS consisted of 200 bursts, repeated continuously at a $5 \mathrm{~Hz}$ frequency for a total of 600 stimuli in $40 \mathrm{~s}$. Sixty MEPs were collected before iTBS or cTBS (baseline) and at two different time points ( 0 and $15 \mathrm{~min}$ ) after the end of stimulations. Stimulation intensity was set to induce a stable MEP of $\sim 1 \mathrm{mV}$ peak-to-peak amplitude in the relaxed right FDI at baseline and remained unchanged until the end of recordings. MEP amplitudes were then averaged at each time point and normalized to the mean baseline amplitude (Huang et al., 2005; Mori et al., 2011).

Data analysis. Differences in age and single-pulse TMS parameters between different genotypes were analyzed using a one-way ANOVA. Differences in gender between genotype groups were analyzed using a $\chi^{2}$ test. For each paired-pulse TMS experimental intervention, a repeatedmeasures ANOVA with within-subject factor ISI and between-subjects factor genotype was used on normalized peak-to-peak amplitudes of the mean MEPs of each subject. Post hoc paired $t$ tests were applied when necessary. Duncan's test was used to correct for multiple comparisons. In all figures, error bars refer to the SE.

\section{Results}

\section{Allele frequencies}

The TMS procedure was well tolerated by all subjects. With respect to the SNP rs222747, the study sample was divided in three groups according to alleles "GG," "CG," and "CC." Similarly, the sample population was divided in three groups ("CC," "CT," and "TT") for the SNP rs222749. Allele frequencies of the two SNPs of the TRPV1 gene are shown in Table 1. The allele frequencies found in our sample were similar to HapMap studies in the CEU population (European-Americans; http://hapmap.ncbi.nlm.nih. gov/index.html.en). 


\begin{tabular}{|c|c|c|c|c|c|}
\hline Subjects & Age (years) & $\begin{array}{l}\text { Gender } \\
\text { (female) }\end{array}$ & $\begin{array}{l}\text { RMT } \\
(\% \text { MSO) }\end{array}$ & $\begin{array}{l}\text { AMT } \\
\text { (\% MSO) }\end{array}$ & $\begin{array}{l}\text { MEP } 1 \mathrm{mV} \\
\text { (\% MSO) }\end{array}$ \\
\hline \multicolumn{6}{|l|}{ rs 222747} \\
\hline GG & $36.0 \pm 8.1$ & $60 \%$ & $43.8 \pm 8.9$ & $37.5 \pm 7.2$ & $61.4 \pm 9.3$ \\
\hline CG & $39.1 \pm 10.1$ & $49 \%$ & $45.8 \pm 6.7$ & $33.3 \pm 5.5$ & $57.6 \pm 8.9$ \\
\hline$C C$ & $39.4 \pm 10.6$ & $40 \%$ & $46.4 \pm 7.2$ & $35.3 \pm 8.5$ & $58.7 \pm 11.3$ \\
\hline \multicolumn{6}{|l|}{ rs222749 } \\
\hline $\mathbb{C C}$ & $38.8 \pm 10.4$ & $53 \%$ & $47.4 \pm 9.2$ & $36.0 \pm 8.3$ & $59.4 \pm 7.3$ \\
\hline CT & $40.6 \pm 12.0$ & $55 \%$ & $43.8 \pm 8.2$ & $29.0 \pm 8.0$ & $56.6 \pm 8.4$ \\
\hline $\mathrm{TT}$ & $42.0 \pm 13.3$ & $40 \%$ & $52.0 \pm 11.2$ & $39.3 \pm 9.5$ & $60.1 \pm 10.2$ \\
\hline
\end{tabular}

MSO, Maximal stimulator output.

\section{SNP rs222747}

No differences in age and sex between genotypes emerged by using, respectively, a one-way ANOVA and $\chi^{2}$ tests.

Mean RMT, AMT, and stimulus intensity required to elicit an MEP of $1 \mathrm{mV}$ amplitude were not significantly different among GG, CG, and CC subjects (Table 2).

We also addressed the possible role of rs 222747 in the regulation of synaptic excitability explored by means of paired-pulse TMS experiments. Analysis was performed using a repeatedmeasures ANOVA design on the normalized data with genotype as between-subjects and ISI as within-subject main factors. The analysis showed a significant main effect of ISI $(F=21.8, p<$ $0.01)$ and genotype $(F=3.0, p<0.05)$ and a significant genotype $\times$ ISI interaction $(F=1.8, p<0.05)$ on SICF values. Post hoc contrasts revealed that SICF elicited in the GG group was significantly larger than in the other two groups at ISIs 1.5 and $2.7 \mathrm{~ms}$. SICI, ICF, and LICI showed a significant effect of ISI $(F=8.8, p<$ 0.01 for SICI; $F=10.3, p<0.01$ for ICF; and $F=11.1, p<0.01$ for LICI) but no significant effect of genotype and genotype $\times$ ISI interaction (Fig. $1 A-C$ ).

In iTBS experiments, we tested whether the rs 222747 polymorphism of the TRPV1 gene affected LTP-like synaptic plasticity. ANOVA on the normalized data revealed a significant effect of time $(F=4.5, p<0.05)$. Conversely, genotype and genotype $\times$ time interaction were not significant (Fig. $1 D$ ).

\section{SNP rs222749}

No differences in age and sex between genotypes emerged by using, respectively, a one-way ANOVA and $\chi^{2}$ tests. Mean RMT, $\mathrm{AMT}$, and stimulus intensity required to elicit an MEP of $1 \mathrm{mV}$ amplitude were not significantly different among CC, CT, and TT subjects (Table 2).

SICF, SICI, ICF, and LICI showed a significant effect of ISI $(F=3.2, p<0.05$ for SICF; $F=6.5, p<0.01$ for SICI; $F=3.7$, $p<0.05$ for ICF; and $F=8.3, p<0.01$ for LICI). Unlike SNP rs222747, no differences were found on SICF, SICI/ICF, and LICI among the CC, CT, and TT subjects (Fig. $2 A-C$ ). Also in the plasticity experiments, the normalized data revealed a significant main effect of time $(F=7.6, p<0.05)$ on the MEP size in response to iTBS, whereas effects of genotype and genotype $\times$ time interaction were not significantly different among CC, CT, and TT subjects (Fig. 2D).

\section{Role of TRPV1 rs222747 SNP on intrinsic excitability and LTD-like plasticity}

The results above indicate that TRPV1 rs222747 SNP could affect glutamate transmission within the CNS. It is thought that SICF at each peak reflects a facilitatory interaction of cortical circuits that are also involved in the generation of distinct indirect (I)-waves in response to transcranial stimulation of the motor cortex (Tokimura et al., 1996; Ziemann et al., 1998a,b; Rothwell, 1999). Spinal epidural recordings show that the amplitude of the earliest I-wave (I1) evoked by TMS can be selectively decreased by an inhibitory form of TBS (cTBS) (Di Lazzaro et al., 2005), whereas iTBS increases MEPs, through an increase in the amplitude of later I-waves but not the I1-wave (Di Lazzaro et al., 2008). We thus explored the effects of this SNP on other measures of cortical excitability to further address the specificity of the observed effect. A recruitment curve and cTBS protocols were performed in a subgroup of 21 (seven CC, seven CG, and seven GG) subjects to evaluate the potential involvement of TRPV1 channels in the control of intrinsic neuronal excitability or LTD-like plasticity. ANOVA on the normalized data revealed a significant effect of intensity for recruitment curves and of time for cTBS $(F=4.5$, $p<0.05)$. Conversely, genotype, genotype $\times$ intensity, and genotype $\times$ time interactions were not significant (Fig. 3).

\section{Discussion}

TRPV1 channels have been described in the dorsal root ganglia and peripheral sensory nerve endings, in which they participate in the detection of noxious chemical and thermal stimuli. Capsaicin (from red pepper) is a well known agonist of TRPV1 channels. Activation of TRPV1 channels on sensory neurons causes the release of glutamate and of pro-inflammatory neuropeptide substance $\mathrm{P}$ and calcitonin G-related peptide in the dorsal horn of the spinal cord, which are critical for transmitting pain signals from the periphery to the CNS (Jara-Oseguera et al., 2008; Premkumar, 2010). TRPV1 channels also participate in the control of synaptic transmission in a variety of central structures, which include the cortex, the hippocampus, the cerebellum, and the basal ganglia (Yang et al., 1998; Mezey et al., 2000; Marinelli et al., 2002, 2003; Li et al., 2004; Derbenev et al., 2006; Starowicz et al., 2007; Xing and Li, 2007; Musella et al., 2009). However, despite intensive research, the function of central TRPV1 channels is only beginning to be understood (Mezey et al., 2000; Cristino et al., 2006; Tzavara et al., 2006; Di Marzo and Cristino, 2008; Maccarrone et al., 2008).

In the present study, we wanted to investigate how two common SNPs of the TRPV1 gene (rs222747 and rs222749) affect cortical excitability in humans. Notably, SNP rs222747 variants carrying the $\mathrm{G}$ allele have been reported to cause greater maximal TRPV1 channel response to capsaicin and to its natural agonist anandamide and to increase TRPV1 mRNA and protein expression in cell surface (Xu et al., 2007). Variants of rs222749, conversely, did not result in apparent significant changes of channel activity (Xu et al., 2007). However, the limited number of subjects bearing the TT genotype of rs222749, indeed very uncommon in the general population and in our series ( 5 of 550 subjects), prevents us from drawing final conclusions on the potential impact of this SNP in the regulation of cortical excitability. In contrast, the results related to the rs222747 SNP seem to be robust enough to justify the conclusion that the TRPV1 channels regulate cortical excitability in the human cortex, because those subjects homozygous for the G allele of this SNP had larger SICF than CG and CC subjects. Conversely, no differences were observed between the three allele groups with regard to the other single- and paired-pulse TMS paradigms and to TBS responses.

TMS delivered on M1 generates high-frequency repetitive discharges of corticospinal cells with a periodicity of $1.5 \mathrm{~ms}$, which are called I-waves (Ziemann and Rothwell, 2000). Because the three peaks of SICF also occur at $\sim 1.5 \mathrm{~ms}$ intervals, SICF is thought to be determined by the summation of the I-waves gen- 
erated by two consecutive TMS pulses separated by ISIs of 1.1-1.5, 2.3-2.9, and 4.1-4.5 $\mathrm{ms}$ on the corticospinal neurons (Ziemann et al., 1998b). Animal experiments provided evidence that I-waves are generated trans-synaptically through excitatory corticocortical fibers (Amassian et al., 1987). SICF peaks are thought to reflect a facilitatory interaction between the effects of the two stimuli at the axon initial segments of the excitatory interneurons in motor cortex, which contribute to the generation of I-waves (Hanajima et al., 2002). The magnitude of these peaks may reflect the strength of excitatory synaptic interactions in M1.

Because I-waves have a trans-synaptic origin, it is hypothesized that most of the EPSPs that cause I-waves might be transmitted by glutamate (Amassian et al., 1987; Ziemann et al., 1998b; Di Lazzaro et al., 2003). Conversely, SICI and ICF likely result from recruitment of different intracortical circuits able to modulate the corticospinal output in the M1. SICI is in part mediated by GABA via postsynaptic $\mathrm{GABA}_{\mathrm{A}}$ receptors (Florian et al., 2008), whereas ICF involves excitatory connections to pyramidal neurons (Kujirai et al., 1993) and LICI very likely reflects $G_{A B A}$ receptor activation as suggested by data from healthy volunteers exposed to baclofen, a $\mathrm{GABA}_{\mathrm{B}}$ receptor agonist (McDonnell et al., 2006; Florian et al., 2008; Vahabzadeh-Hagh et al., 2011). Recruitment curves are considered sensitive measures of changes in neuronal system intrinsic excitability. Unlike SICF, recruitment curves were not affected by rs222747 TRPV1 SNP. Previous work showed they are influenced by norepinephrine and serotonin levels (Plewnia et al., 2001, 2002; Gerdelat-Mas et al., 2005), suggesting the idea that the effect of this SNP on synaptic transmission is rather selective.

Although our results may be consistent with the idea that enhancement of glutamatergic transmission underlies the observed effect on SICF of the rs222747 GG genotype, it is noteworthy that other TMS parameters not altered by rs222747 SNP, such as RMT, MEP amplitude, ICF, and LTP- and LTD-like plasticity, have been postulated to be also glutamate dependent (Liepert et al., 1997; Schwenkreis et al., 2000; Di Lazzaro et al., 2003; Reis et al., 2006; Huang et al., 2007, 2008). However, previous studies aimed at exploring the TMS effects of a variety of glutamatergic drugs found modulatory effects only in one or few of these parameters, depending on the specific mechanism of action of the drug under investigation (Liepert et al., 1997; Schwenkreis et al., 2000; Di Lazzaro et al.,
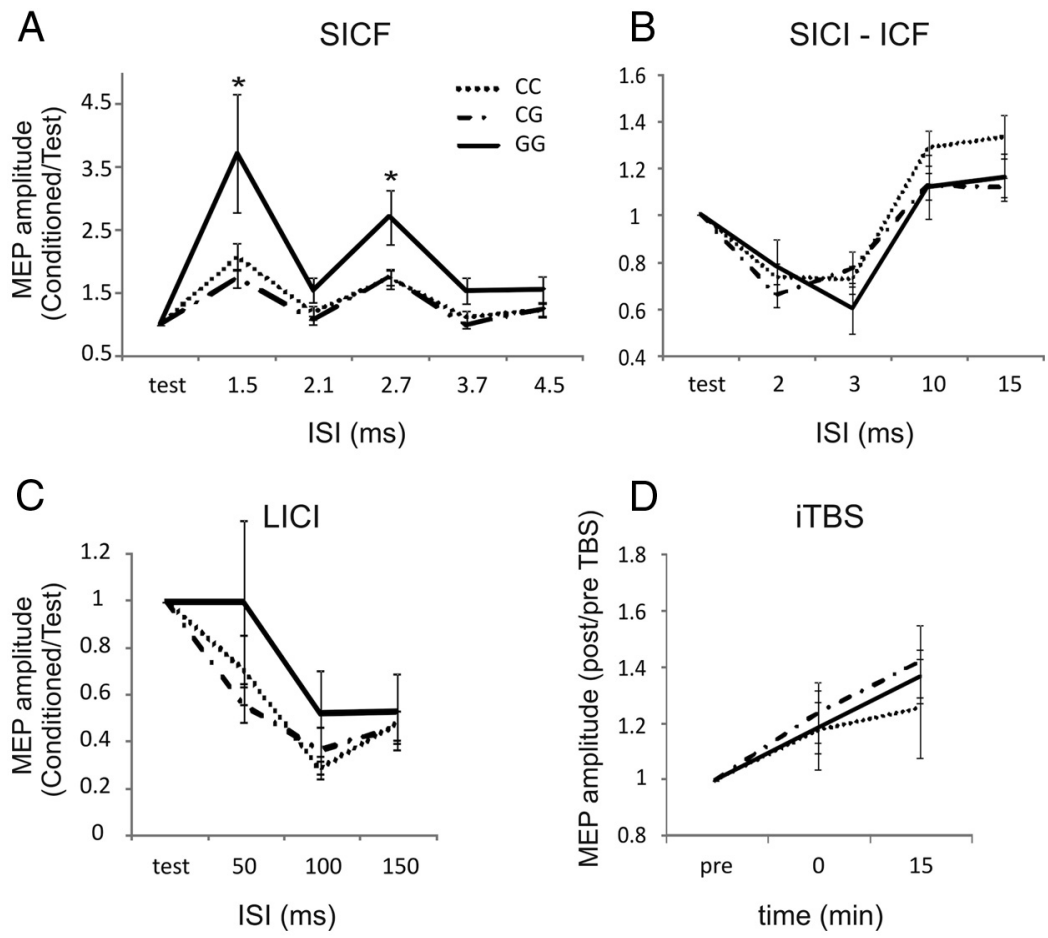

Figure 1. Roleofrs222747 polymorphismintheregulation of cortical excitability.A, In subjects carnying the GGallele,SICFwassignificantlylarger than in the other subjects atall ISIs. No significant effect was found on SICI/CF (B), LCI (C), andiTBS-induced plasticity (D). In $A-C$, thex-axis indicates the IS I in milliseconds, and they-axis shows the MEP size elicited by paired-pulse stimulation.
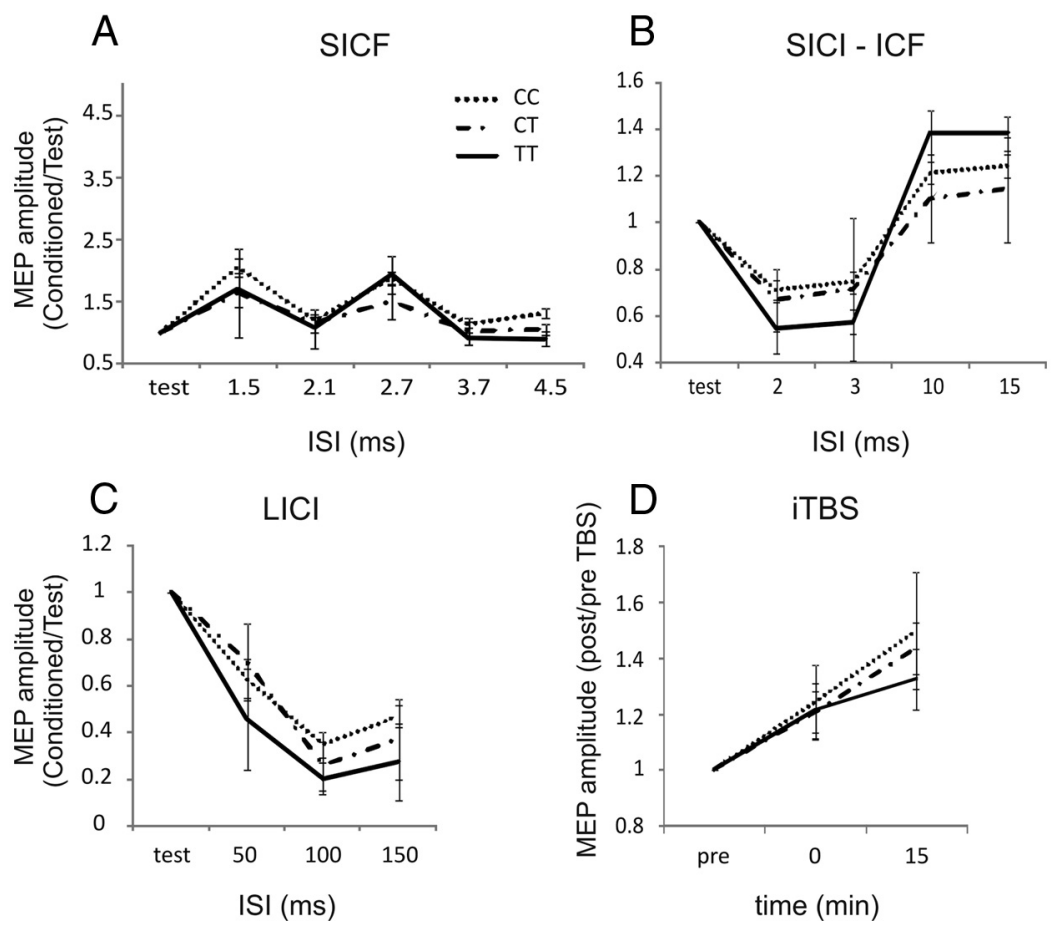

Figure 2. Role of rs 222749 polymorphism in the regulation of cortical excitability. No differences were found between $C C, C T$, and TT individuals in the SICF $(\boldsymbol{A})$, SICI/ICF $(\boldsymbol{B}), \mathrm{LICI}(\boldsymbol{C})$, and iTBS-induced plasticity (D). As in Figure 1, the $x$-axis of $\boldsymbol{A}$-Cindicates the ISI in milliseconds, and the $y$-axis shows the MEP size elicited by paired-pulse stimulation.

2003; Reis et al., 2006). Based on these premises, it is therefore conceivable that TRPV1 effects on glutamate transmission are not widespread but are rather selective for the synaptic underpinnings of SICF generation. Glutamate transmission can be modu- 

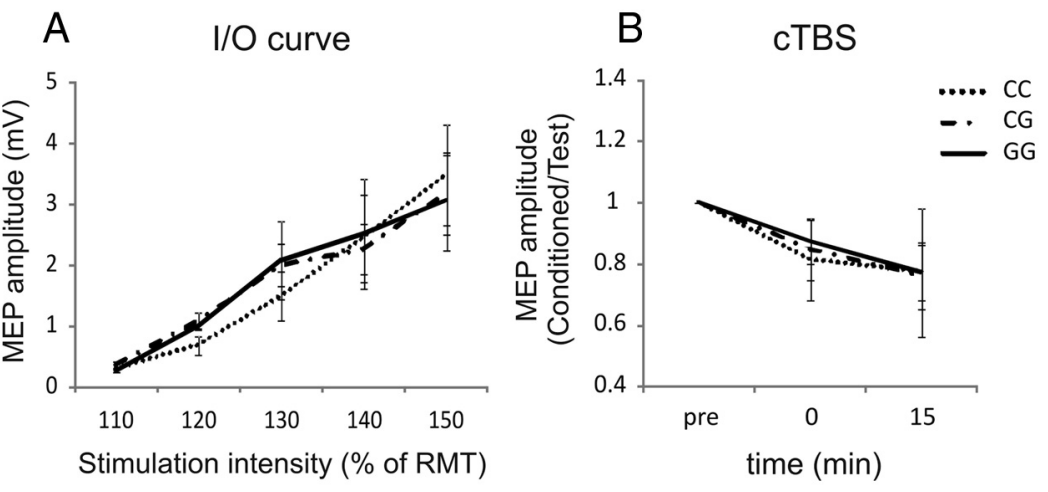

Figure 3. Role of rs 222747 polymorphism in the regulation of intrinsic neuronal excitability and LTD-like plasticity. No differences were found in the recruitment $(\mathrm{I} / 0)$ curves $(\boldsymbol{A})$ and in the responses to $\mathrm{CTBS}(\boldsymbol{B})$ between $\mathrm{GG}, \mathrm{CG}$, and $C($ subjects. I/O curve, Input/output curve.

lated by presynaptic or postsynaptic effects, and TRPV1 channels are rather selectively involved in the modulation of glutamate transmission by favoring neurotransmitter release from nerve endings. Interestingly, a short-term, purely presynaptic form of synaptic potentiation, known as paired-pulse facilitation (PPF), is evoked in in vitro preparations by coupling two stimuli with short ISIs (Thomson, 2000). TRPV1 channels have been shown to significantly influence PPF in vitro through a presynaptic mechanism (Derbenev et al., 2006). Along with the results of the present investigation, this information may therefore suggest that the SNP rs222747 effects on SICF reflect the presynaptic action of TRPV1 channels on glutamatergic terminals. In this respect, even if PPF and SICF cannot be directly compared, because PPF is attributed to the presynapse but for SICF it is less clear whether it is a presynaptic or postsynaptic phenomenon, the SICF protocol is reminiscent of the in vitro PPF paradigm, and a similar incremental effect on SICF without changes in other single- and paired-pulse TMS measures was observed in healthy subjects after intake of subanesthetic doses of the NMDA antagonist ketamine (Di Lazzaro et al., 2003). Low doses of ketamine produce a dual modulating effect on glutamatergic transmission in experimental animals, blocking NMDA receptor activity and enhancing non-NMDA transmission through an increase in the release of endogenous glutamate (Bustos et al., 1992). Thus, our hypothesis is that enhancing TRPV1 channel function in the SNP rs222747 GG subjects may influence cortical excitability through an increase of glutamate transmission. Several data have shown that activation of TRPV 1 channels potentiates glutamate release from excitatory terminals, although their effects on GABA release are less consistent (Yang et al., 1998; Marinelli et al., 2002, 2003; Li et al., 2004; Derbenev et al., 2006; Starowicz et al., 2007; Xing and Li, 2007; Musella et al., 2009). It may be argued that none of these animal studies have addressed so far the effect of TRPV1 channels in the cerebral cortex, whose excitability has been explored in our TMS work. However, the remarkable consistency of the effects found in rodent spinal cord (Yang et al., 1998), substantia nigra (Marinelli et al., 2003), locus ceruleus (Marinelli et al., 2002), hypothalamus (Li et al., 2004), nucleus of the vagus nerve (Derbenev et al., 2006), brainstem (Starowicz et al., 2007), dorsolateral periaqueductal gray neurons (Xing and Li, 2007), and striatum (Musella et al., 2009) strongly suggests that the synaptic effect of these channels may be similar also in the cerebral cortex.

The long-lasting facilitatory or inhibitory aftereffects on MEP amplitude in response to TBS and the LTP/LTD of synaptic trans585:105-115. mission described in animal studies are likely related phenomena, because blockade of NMDA receptors prevents both forms of synaptic plasticity (Huang et al., 2007, 2008). Notably, SNP variants of the TRPV1 channels failed to affect iTBS and cTBS responses in our study, although the induction of hippocampal LTP/LTD requires the activation of TRPV1 channels (Marsch et al., 2007; Gibson et al., 2008; Peters et al., 2010; Zhou et al., 2010). One possible explanation for this finding is that MEP changes secondary to TBS are already maximal in wild-type SNP rs222747 subjects and cannot be therefore further potentiated by enhancing the function of TRPV1 channels in the homozygous GG genotype. However, it should also be considered that the small sample size could have led to nonsignificant results attributable to lack of statistical power. Finally, direct comparisons between TBS in humans and LTP/LTD in animals must be taken with caution, because it is a matter of ongoing debate whether they share similar mechanisms. Indeed, TBS- and TRPV1-mediated synaptic plasticity may be based on different mechanisms.

Despite these limitations, to our knowledge, our results provide the first evidence that TRPV1 channels regulate synaptic transmission in humans. Additional experimental work in a larger sample of patients and exploring other neurophysiological and functional imaging measures of synaptic excitability are mandatory to extend our results.

\section{References}

Amassian VE, Stewart M, Quirk GJ, Rosenthal JL (1987) Physiological basis of motor effects of a transient stimulus to cerebral cortex. Neurosurgery 20:74-93.

Bustos G, Abarca J, Forray MI, Gysling K, Bradberry CW, Roth RH (1992) Regulation of excitatory amino acid release by $N$-methyl-D-aspartate receptors in rat striatum: in vivo microdialysis studies. Brain Res

Caterina MJ, Schumacher MA, Tominaga M, Rosen TA, Levine JD, Julius D (1997) The capsaicin receptor: a heat-activated ion channel in the pain pathway. Nature 389:816-824.

Cristino L, de Petrocellis L, Pryce G, Baker D, Guglielmotti V, Di Marzo V (2006) Immunohistochemical localization of cannabinoid type 1 and vanilloid transient receptor potential vanilloid type 1 receptors in the mouse brain. Neuroscience 139:1405-1415.

De Petrocellis L, Di Marzo V (2005) Lipids as regulators of the activity of transient receptor potential type V1 (TRPV1) channels. Life Sci 77:16511666.

Derbenev AV, Monroe MJ, Glatzer NR, Smith BN (2006) Vanilloidmediated heterosynaptic facilitation of inhibitory synaptic input to neurons of the rat dorsal motor nucleus of the vagus. J Neurosci 26:9666-9672.

Di Lazzaro V, Oliviero A, Profice P, Pennisi MA, Pilato F, Zito G, Dileone M, Nicoletti R, Pasqualetti P, Tonali PA (2003) Ketamine increases human motor cortex excitability to transcranial magnetic stimulation. J Physiol 547:485-496.

Di Lazzaro V, Pilato F, Saturno E, Oliviero A, Dileone M, Mazzone P, Insola A, Tonali PA, Ranieri F, Huang YZ, Rothwell JC (2005) Theta-burst repetitive transcranial magnetic stimulation suppresses specific excitatory circuits in the human motor cortex. J Physiol 565:945-950.

Di Lazzaro V, Pilato F, Dileone M, Profice P, Oliviero A, Mazzone P, Insola A, Ranieri F, Meglio M, Tonali PA, Rothwell JC (2008) The physiological basis of the effects of intermittent theta burst stimulation of the human motor cortex. J Physiol 586:3871-3879.

Di Marzo V, Cristino L (2008) Why endocannabinoids are not all alike. Nat Neurosci 11:124-126. 
Di Marzo V, De Petrocellis L (2010) Endocannabinoids as regulators of transient receptor potential (TRP) channels: a further opportunity to develop new endocannabinoid-based therapeutic drugs. Curr Med Chem 17:1430-1449.

Florian J, Müller-Dahlhaus M, Liu Y, Ziemann U (2008) Inhibitory circuits and the nature of their interactions in the human motor cortex a pharmacological TMS study. J Physiol 586:495-514.

Gerdelat-Mas A, Loubinoux I, Tombari D, Rascol O, Chollet F, SimonettaMoreau M (2005) Chronic administration of selective serotonin reuptake inhibitor (SSRI) paroxetine modulates human motor cortex excitability in healthy subjects. Neuroimage 27:314-322.

Gibson HE, Edwards JG, Page RS, Van Hook MJ, Kauer JA (2008) TRPV1 channels mediate long-term depression at synapses on hippocampal interneurons. Neuron 57:746-759.

Hanajima R, Ugawa Y, Terao Y, Enomoto H, Shiio Y, Mochizuki H, Furubayashi T, Uesugi H, Iwata NK, Kanazawa I (2002) Mechanisms of intracortical I-wave facilitation elicited with paired-pulse magnetic stimulation in humans. J Physiol 538:253-261.

Huang YZ, Edwards MJ, Rounis E, Bhatia KP, Rothwell JC (2005) Theta burst stimulation of the human motor cortex. Neuron 45:201-206.

Huang YZ, Chen RS, Rothwell JC, Wen HY (2007) The after-effect of human theta burst stimulation is NMDA receptor dependent. Clin Neurophysiol 118:1028-1032.

Huang YZ, Rothwell JC, Edwards MJ, Chen RS (2008) Effect of physiological activity on an NMDA-dependent form of cortical plasticity in human. Cereb Cortex 18:563-570.

Jara-Oseguera A, Simon SA, Rosenbaum T (2008) TRPV1: on the road to pain relief. Curr Mol Pharmacol 1:255-269.

Jurinke C, van den Boom D, Cantor CR, Köster H (2002) Automated genotyping using the DNA MassArray technology. Methods Mol Biol 187:179-192.

Kujirai T, Caramia MD, Rothwell JC, Day BL, Thompson PD, Ferbert A, Wroe S, Asselman P, Marsden CD (1993) Corticocortical inhibition in human motor cortex. J Physiol 471:501-519.

Li DP, Chen SR, Pan HL (2004) VR1 receptor activation induces glutamate release and postsynaptic firing in the paraventricular nucleus. J Neurophysiol 92:1807-1816.

Liepert J, Schwenkreis P, Tegenthoff M, Malin JP (1997) The glutamate antagonist riluzole suppresses intracortical facilitation. J Neural Transm 104:1207-1214.

Maccarrone M, Rossi S, Bari M, De Chiara V, Fezza F, Musella A, Gasperi V, Prosperetti C, Bernardi G, Finazzi-Agrò A, Cravatt BF, Centonze D (2008) Anandamide inhibits metabolism and physiological actions of 2-arachidonoylglycerol in the striatum. Nat Neurosci 11:152-159.

Marinelli S, Vaughan CW, Christie MJ, Connor M (2002) Capsaicin activation of glutamatergic synaptic transmission in the rat locus coeruleus in vitro. J Physiol 543:531-540.

Marinelli S, Di Marzo V, Berretta N, Matias I, Maccarrone M, Bernardi G, Mercuri NB (2003) Presynaptic facilitation of glutamatergic synapses to dopaminergic neurons of the rat substantia nigra by endogenous stimulation of vanilloid receptors. J Neurosci 23:3136-3144.

Marsch R, Foeller E, Rammes G, Bunck M, Kössl M, Holsboer F, Zieglgänsberger W, Landgraf R, Lutz B, Wotjak CT (2007) Reduced anxiety, conditioned fear, and hippocampal long-term potentiation in transient receptor potential vanilloid type 1 receptor-deficient mice. J Neurosci $27: 832-839$.

McDonnell MN, Orekhov Y, Ziemann U (2006) The role of GABA(B) receptors in intracortical inhibition in the human motor cortex. Exp Brain Res 173:86-93.

Mezey E, Tóth ZE, Cortright DN, Arzubi MK, Krause JE, Elde R, Guo A, Blumberg PM, Szallasi A (2000) Distribution of mRNA for vanilloid receptor subtype 1 (VR1), and VR1-like immunoreactivity, in the central nervous system of the rat and human. Proc Natl Acad Sci U S A 97:3655-3660.

Mori F, Rossi S, Sancesario G, Codecà C, Mataluni G, Monteleone F, Buttari F, Kusayanagi H, Castelli M, Motta C, Studer V, Bernardi G, Koch G, Bernardini S, Centonze D (2011) Cognitive and cortical plasticity deficits correlate with altered amyloid- $\beta$ CSF levels in multiple sclerosis. Neuropsychopharmacology 36:559-568.

Musella A, De Chiara V, Rossi S, Prosperetti C, Bernardi G, Maccarrone M, Centonze D (2009) TRPV1 channels facilitate glutamate transmission in the striatum. Mol Cell Neurosci 40:89-97.

Oldfield RC (1971) The assessment and analysis of handedness: the Edinburgh inventory. Neuropsychologia 9:97-113.
Peters JH, McDougall SJ, Fawley JA, Smith SM, Andresen MC (2010) Primary afferent activation of thermosensitive TRPV1 triggers asynchronous glutamate release at central neurons. Neuron 65:657-669.

Peurala SH, Müller-Dahlhaus JF, Arai N, Ziemann U (2008) Interference of short-interval intracortical inhibition (SICI) and short-interval intracortical facilitation (SICF). Clin Neurophysiol 119:2291-2297.

Pingle SC, Matta JA, Ahern GP (2007) Capsaicin receptor: TRPV1 a promiscuous TRP channel. Handb Exp Pharmacol 179:155-171.

Plewnia C, Bartels M, Cohen L, GerloffC (2001) Noradrenergic modulation of human cortex excitability by the presynaptic alpha(2)-antagonist yohimbine. Neurosci Lett 307:41-44.

Plewnia C, Hoppe J, Hiemke C, Bartels M, Cohen LG, Gerloff C (2002) Enhancement of human cortico-motoneuronal excitability by the selective norepinephrine reuptake inhibitor reboxetine. Neurosci Lett 330:231-234.

Premkumar LS (2010) Targeting TRPV1 as an alternative approach to narcotic analgesics to treat chronic pain conditions. AAPS J 12:361-370.

Reis J, John D, Heimeroth A, Mueller HH, Oertel WH, Arndt T, Rosenow F (2006) Modulation of human motor cortex excitability by single doses of amantadine. Neuropsychopharmacology 31:2758-2766.

Rothwell JC (1997) Techniques and mechanisms of action of transcranial stimulation of the human motor cortex. J Neurosci Methods 74:113-122.

Rothwell JC (1999) Paired-pulse investigations of short-latency intracortical facilitation using TMS in humans. Electroencephalogr Clin Neurophysiol Suppl 51:113-119.

Schwenkreis P, Liepert J, Witscher K, Fischer W, Weiller C, Malin JP, Tegenthoff M (2000) Riluzole suppresses motor cortex facilitation in correlation to its plasma level. A study using transcranial magnetic stimulation. Exp Brain Res 135:293-299.

Shoudai K, Peters JH, McDougall SJ, Fawley JA, Andresen MC (2010) Thermally active TRPV1 tonically drives central spontaneous glutamate release. J Neurosci 30:14470-14475.

Sikand P, Premkumar LS (2007) Potentiation of glutamatergic synaptic transmission by protein kinase C-mediated sensitization of TRPV1 at the first sensory synapse. J Physiol 581:631-647.

Starowicz K, Maione S, Cristino L, Palazzo E, Marabese I, Rossi F, de Novellis V, Di Marzo V (2007) Tonic endovanilloid facilitation of glutamate release in brainstem descending antinociceptive pathways. J Neurosci 27:13739-13749.

Suh YG, Oh U (2005) Activation and activators of TRPV1 and their pharmaceutical implication. Curr Pharm Des 11:2687-2698.

Thomson AM (2000) Facilitation, augmentation and potentiation at central synapses. Trends Neurosci 23:305-312.

Tokimura H, Ridding MC, Tokimura Y, Amassian VE, Rothwell JC (1996) Short latency facilitation between pairs of threshold magnetic stimuli applied to human motor cortex. Electroencephalogr Clin Neurophysiol 101:263-272.

Tzavara ET, Li DL, Moutsimilli L, Bisogno T, Di Marzo V, Phebus LA, Nomikos GG, Giros B (2006) Endocannabinoids activate transient receptor potential vanilloid 1 receptors to reduce hyperdopaminergia-related hyperactivity: therapeutic implications. Biol Psychiatry 59:508 -515.

Vahabzadeh-Hagh AM, Muller PA, Pascual-Leone A, Jensen FE, Rotenberg A (2011) Measures of cortical inhibition by paired-pulse transcranial magnetic stimulation in anesthetized rats. J Neurophysiol 105:615-624.

Valls-Solé J, Pascual-Leone A, Wassermann EM, Hallett M (1992) Human motor evoked responses to paired transcranial magnetic stimuli. Electroencephalogr Clin Neurophysiol 85:355-364.

Van Der Stelt M, Di Marzo V (2004) Endovanilloids. Putative endogenous ligands of transient receptor potential vanilloid 1 channels. Eur J Biochem 271:1827-1834.

van der Stelt M, Di Marzo V (2005) Anandamide as an intracellular messenger regulating ion channel activity. Prostaglandins Other Lipid Mediat 77:111-122.

Werhahn KJ, Kunesch E, Noachtar S, Benecke R, Classen J (1999) Differential effects on motorcortical inhibition induced by blockade of GABA uptake in humans. J Physiol 517:591-597.

Xing J, Li J (2007) TRPV1 receptor mediates glutamatergic synaptic input to dorsolateral periaqueductal gray (dl-PAG) neurons. J Neurophysiol 97:503-511.

Xu H, Tian W, Fu Y, Oyama TT, Anderson S, Cohen DM (2007) Functional effects of nonsynonymous polymorphisms in the human TRPV1 gene. Am J Physiol Renal Physiol 293:F1865-F1876. 
Yang K, Kumamoto E, Furue H, Yoshimura M (1998) Capsaicin facilitates excitatory but not inhibitory synaptic transmission in substantia gelatinosa of the rat spinal cord. Neurosci Lett 255:135-138.

Zhou HY, Chen SR, Chen H, Pan HL (2010) Opioid-induced long-term potentiation in the spinal cord is a presynaptic event. J Neurosci 30:4460-4466.

Ziemann U (2004) TMS and drugs. Clin Neurophysiol 115:1717-1729.

Ziemann U, Rothwell JC (2000) I-waves in motor cortex. J Clin Neurophysiol 17:397-405.
Ziemann U, Tergau F, Wassermann EM, Wischer S, Hildebrandt J, Paulus W (1998a) Demonstration of facilitatory I waves interaction in the human motor cortex by paired transcranial magnetic stimulation. J Physiol $511: 181-190$

Ziemann U, Tergau F, Wischer S, Hildebrandt J, Paulus W (1998b) Pharmacological control of facilitatory I-wave interaction in the human motor cortex. A paired transcranial magnetic stimulation study. Electroencephalogr Clin Neurophysiol 109:321-330. 